\title{
Bipolar charge transport model to estimate charging and degradation processes in electron-beam irradiated LDPE films
}

\author{
M E Banda, S Le Roy, V Griseri, and G Teyssèdre \\ LAPLACE, Université de Toulouse, CNRS, INPT, UPS, France \\ Email: banda@laplace.univ-tlse.fr
}

\begin{abstract}
Improvement of the performances of dielectric materials subjected to electrical stresses is one of the challenging objectives in a large number of domains of industrial applications. Achieving this goal requires a better understanding of the physical processes governing the behavior of dielectrics, and on their long term degradation. The present paper proposes the coupling of a fluidtype of charge transport model to reproduce the space charge behavior and original experimental data using electron beam irradiation to try to unravel the contributions of charging processes from material degradation in the dielectric response. The charge transport model has been developed using Comsol Multiphysics $\odot$ and adapted to electron beam irradiation. Hence it takes into account the physical processes linked to e-beam irradiation. The model globally reproduces most of the experimental characteristics measured experimentally. It seems that the specific behavior observed experimentally comes from space charge, although conclusions on the model parameterization show that chemical modification could also take place.
\end{abstract}

PACS Numbers: $72.20 \mathrm{Ht}, 72.20 \mathrm{Jv}, 77.22 \mathrm{Ej}, 77.22 \mathrm{Jp}$

Keywords- Fluid model, Electron-beam irradiation, Space charge, Insulating materials, degradation.

\section{Introduction}

Modeling charge generation and transport phenomena constitutes a complementary way to experimental measurements to improve the understanding of charge accumulation and ageing mechanisms in organic materials used as electrical insulators in different domains of application. This study shows a new approach through the simulation of charge transport processes induced by charges generated by electron-beam deposition and characterized in post-irradiation using high DC stress. This approach should help understanding the complex response measured experimentally for electron beam irradiated low density polyethylene (LDPE) samples characterized using space charge and current measurements [1]. A fluid model [2-4] has been developed using Finite Elements Modeling (FEM) tool (Comsol Multiphysics()) and adapted to electron-beam irradiation [5, 6]. It takes into account electronic charge generation at the electrodes, bipolar transport, trapping and detrapping into deep traps, and charge recombination. It also includes physical processes directly related to electron-beam irradiation. The development of such model using FEM is motivated by the opportunity to easily develop multidimensional models in the future, on the basis of the one dimension model developed in this paper. The first objective is for the model to reproduce the different specific behaviors observed experimentally, under irradiation and after irradiation. The second objective is to highlight, with the help of the model, the main physical processes that dominate the space charge and current behavior, 
when a LDPE is subjected to electron-beam irradiation and whether the sample has undergone chemical degradation or not.

\section{Experimental procedure and model formulation}

\subsection{Material and experimental configurations}

LDPE has been chosen as reference material, as it has a simple chemical structure and transport parameters are relatively well defined from previous works. LDPE samples of $300 \mu \mathrm{m}$ in thickness have been prepared from polyethylene pellets using a hydraulic press. They are then inserted in an irradiation chamber, under a vacuum of $\sim 10^{-7} \mathrm{mbar}$, and submitted to a $80 \mathrm{keV}$ electron-beam with a current flux of $1 \mathrm{nA} / \mathrm{cm}^{2}$, for $10 \mathrm{~min}$. Space charge distributions are probed either under irradiation, using the in-situ Pulsed Electro-Acoustic (PEA) set-up [7], or in post-irradiation. For the later case, samples are removed from the irradiation chamber, i.e. they return to atmospheric conditions, and they are then characterized by 'classic' measurements under applied DC field of $40 \mathrm{kV} / \mathrm{mm}$. Space charge measurements conditions under irradiation and during post-irradiation have already been published [1], and are coupled to current measurements to develop the physical hypotheses included in the model at first, and then to validate the simulation results. Here current measurements were realized for field in the range 5 to $100 \mathrm{kV} / \mathrm{mm}$ on samples provided with $20 \mathrm{~cm}^{2}$ gold electrodes deposited by sputtering after samples irradiation.

\subsection{Model description}

The charge transport model developed in this study is based on [2-4] and features bipolar charge transport with average effective properties. The model takes into account injection of electronic carriers (electrons and holes), conduction through a hopping type mobility function of the electric field and temperature, trapping, detrapping into deep traps and recombination, function of the charge mobility (i.e. Langevin), as illustrated in Figure 1. Processes linked to electron beam irradiation follow the "generation-recombination" model [5-6], i.e. primary electrons deposition (numbered 1 in Figure 1) and electron/hole pairs generation due to the dose (numbered 2 in Figure 1) are also included in the fluid model. Charges can be injected at the electrodes, the sign of the injected charge being dependent upon the electric field inside the material (numbered 3 in Figure 1).

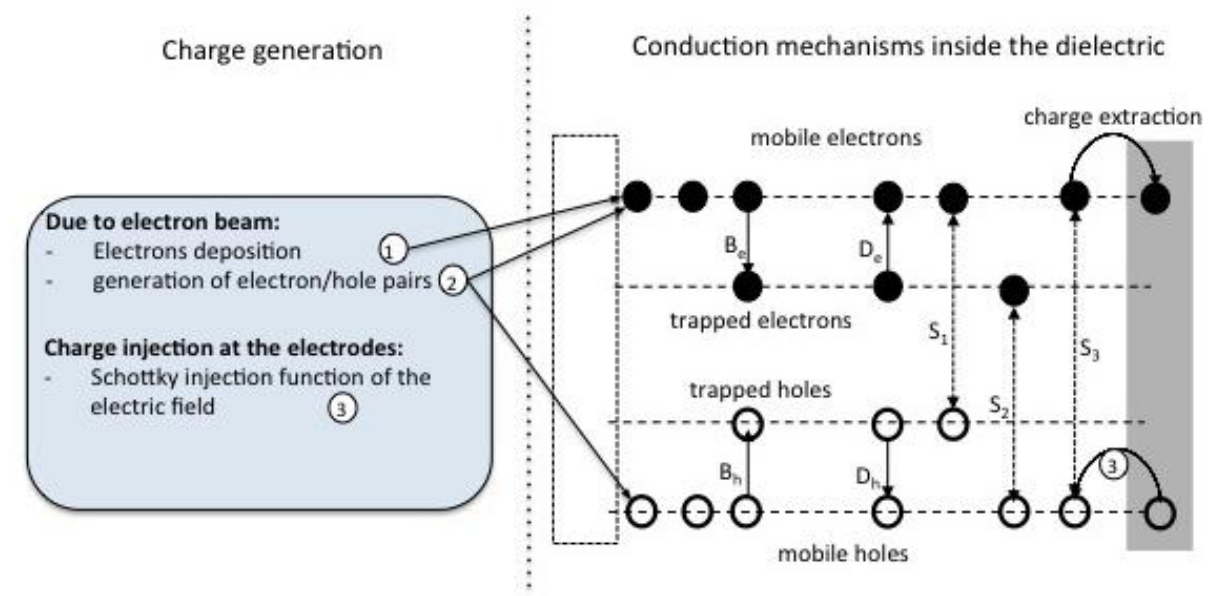

Figure 1. Schematic description of the fluid model applied to electron-beam irradiated LDPE samples.

Whatever the hypotheses considered, the charge transport problem in solid insulators is generally described by the continuity equation (advection equation) coupled with Poisson's equation. In onedimension (1D), according to the spatial coordinate $x$, corresponding to the position inside material thickness, the problem is formulated by:

- Continuity equation, solved for each carrier, mobile or trapped: 


$$
\frac{n_{e, h}(x, t)}{t}+\frac{j_{e, h}(x, t)}{x}=s_{i}(x, t)
$$

- Poisson's equation, expressing the electric field distribution inside the material as a function of net charge density:

$$
\frac{E(x, t)}{x}=\frac{(x, t)}{}
$$

- Transport equation, giving the instantaneous local current density for each transported species, neglecting diffusion:

$$
j_{e, h}(x, t)=n_{e, h}(x, t) \times{ }_{e, h}(E, t) \times E(x, t)
$$

For each equation, the subscripts e and $\mathrm{h}$ refer to electrons and holes respectively. $j_{e, h}\left[\mathrm{~A} \cdot \mathrm{m}^{-2}\right]$ is the carrier current density, $n_{e, h}\left[\mathrm{C} \cdot \mathrm{m}^{-3}\right]$ the charge density of each carrier, ${ }_{e, h}\left[\mathrm{~m}^{2} \cdot \mathrm{V}^{-1} \cdot \mathrm{s}^{-1}\right]$ the free carriers mobility, $E\left[\mathrm{~V} \cdot \mathrm{m}^{-1}\right]$ the electric field, $S_{i}\left[\mathrm{~A} \cdot \mathrm{m}^{-3}\right]$ the source terms, taking into account all physical phenomena other than those related to the transport: trapping, detrapping, recombination, electrons deposition and electron/hole pairs generation due to the e-beam. [C. $\left.\mathrm{m}^{-3}\right]$ is the net charge density given by the algebraic sum of all carrier densities:

$$
(x, t)=n_{h}(x, t)+n_{h t}(x, t) \quad n_{e}(x, t) \quad n_{e t}(x, t)
$$

Where $n_{e}, n_{e t}, n_{h}, n_{h t}\left[\mathrm{C} . \mathrm{m}^{-3}\right]$ are charge densities for mobile electrons, trapped electrons, mobile holes and trapped holes, respectively.

The hopping mobility of free carriers transporting from site to site is given by [C2]:

$$
\mu_{e, h}(E, t)=\frac{2}{E(x, t)}\left[\exp \left(\frac{e, h}{k_{B} T}\right)\right] \sinh \left(\frac{q E(x, t)}{2 k_{B} T}\right)
$$

Where $[\mathrm{m}]$ is the inter-traps distance, $\left[\mathrm{s}^{-1}\right]$ the attempt-to-escape frequency of trapped carriers set to $k_{B} T / h=6.210^{12}\left[\mathrm{~s}^{-1}\right]$ at room temperature $T[300 \mathrm{~K}] ; k_{B}\left[\mathrm{~J} . \mathrm{K}^{-1}\right]$ and $h[\mathrm{~J} . \mathrm{s}]$ are the Boltzmann's and Planck's constants respectively; $q[\mathrm{C}]$ the elementary charge and ${ }_{e, h}[\mathrm{eV}]$ is the hopping potential barrier for electrons and holes. Different hopping potential barriers were chosen for each charge type in consideration of energy level difference of deep traps between electrons and holes [C3].

The extraction of mobile charges at each electrode is considered without extraction barrier, and corresponds, for electrons as an example, to an extraction current density of the type:

$$
j_{e}(X, t)=n_{\theta \mu}(X, t) \times{ }_{\theta \mu}(E, t) \times E(X, t)
$$

Where X corresponds to the spatial coordinate of one electrode, being 0 or $\mathrm{d}$ (the sample thickness), depending on the carrier type and the electric field at the electrode considered.

The resulting source term is thus formulated, for mobile electrons as an example [8]: 


$$
\begin{aligned}
& S_{e \mu}(x, t)=\underbrace{\frac{\partial J_{0}(x)}{\partial x}}_{\text {electron beam }}+\underbrace{G(x)}_{\begin{array}{c}
\text { electronhole } \\
\text { pairs generation }
\end{array}} \overbrace{-B_{e} \cdot n_{e \mu}(x, t)\left(1-\frac{n_{e t}}{N_{0 . e t}}\right)}^{\text {trapping }}+\underbrace{n_{e t}(x, t) \cdot v \exp \left(-\frac{\phi_{\text {tre }}}{k_{B} T}\right)}_{\text {detrapping }} \\
& -\overbrace{S_{1} \cdot n_{e \mu}(x, t) \cdot n_{h t}(x, t)-S_{3} \cdot n_{e \mu}(x, t) \cdot n_{h \mu}(x, t)}^{\text {recombination }}
\end{aligned}
$$

Where $J_{0}(x)$ refers to the beam current density in the dielectric; $B_{\mathrm{e}}$ is the trapping coefficient for electrons, tre the detrapping barrier height for electrons, $N_{0 \text {.et }}$ corresponds to deep trap density for electrons and $s_{0}$ to $s_{3}$ refer to the recombination coefficients. The same kind of equation would hold for trapped electrons, mobile holes and trapped holes, and are detailed in [9]. The source terms due to irradiation (electrons deposition and electron/hole pairs generation) disappear for post-irradiation conditions.

Recombination between different kinds of species as given in Fig. 1 is considered as a function of their mobility (Langevin type) [6]:

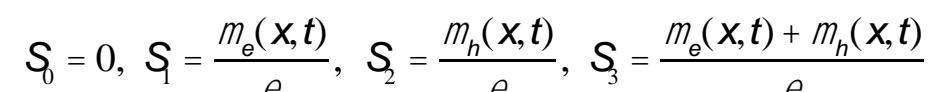

$\mathrm{G}(\mathrm{x})$ is the electron/hole pair generation, given by [6]:

$$
G(x)=G_{0} \cdot D(x)
$$

Where $G_{0}$ is the electron/hole pair generation coefficient. $G(x)$ is considered null in the nonirradiated region (NIR) and primary electrons are considered as a source term for mobile electrons in the model once thermalized. Similarly, carriers issued from electron/hole pairs generation are also considered in the model as initially mobile; $D(x)$ is the normalized dose rate due to the electronbeam, given by:

$$
D(x)=\frac{(x)}{v_{\text {vol }}}\left(\frac{\partial E_{\text {Beam }}(x)}{\partial x}\right)
$$

Where $\left[\mathrm{A} \cdot \mathrm{m}^{-2}\right]$ is the beam current flux, ${ }_{\text {vol }}\left[\mathrm{kg} \cdot \mathrm{m}^{-3}\right]$ the volume density and $E_{\text {Beam }}(X)$ the beam energy in $[\mathrm{eV}]$.

The normalized dose rate, the current density and the electron density due to an electron beam of $80 \mathrm{keV}$ with a constant flux of $1 \mathrm{nA} / \mathrm{cm}^{2}$ in a LDPE sample of thickness $300 \mu \mathrm{m}$ have been calculated using Monte Carlo N-Particle (MCNP) simulation code [10]. This code does not take into account the processes linked to conduction. Figure 2 shows the calculated profiles function of the position inside a LDPE sample when considering a volume density of $0.9 \mathrm{~g} / \mathrm{cm}^{3}$. In these conditions of e-beam irradiation, the maximum deposited dose and electron density are respectively located at around 50 $\mu \mathrm{m}$ and $75 \mu \mathrm{m}$ from the irradiated face, while the irradiated region extends over around $120 \mu \mathrm{m}$. The corresponding averaged deposited dose was estimated around $6 \mathrm{kGy}$. 


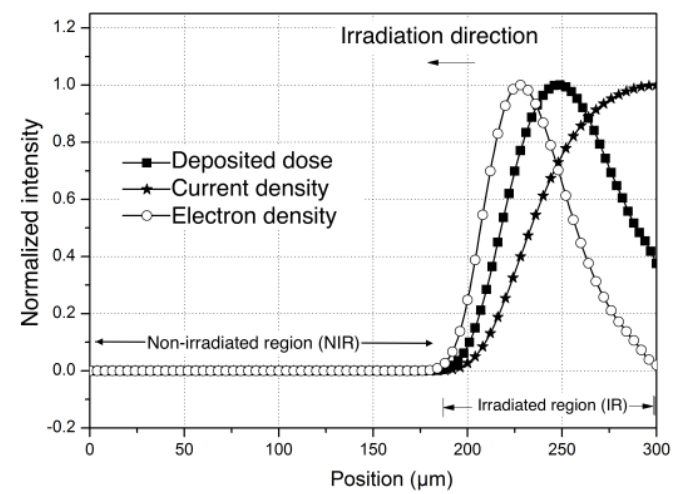

Figure 2. Normalized beam current density, deposited dose and primary electron density due to the electron-beam as a function of the position in the sample. Theoretical calculation performed with MCNP [10] code, for a $300 \mu \mathrm{m}$ thick LDPE irradiated with a $80 \mathrm{keV}$ electron-beam at $1 \mathrm{nA} / \mathrm{cm}^{2}$.

Charge generation at the electrodes follows a modified Schottky law [Alison (review semiconductors), Christen IEEE 2014 on interfaces, Scott and Maliaras (see LeRoy IEEE2016 cylindric to have this reference)], which is based on the hypothesis that the contact is already at a thermodynamic equilibrium prior to any field application, so that the back current coming from the dielectric directed towards the electrode completely cancels the injection current coming from the electrode towards the material:

$$
j_{\text {Schot }}(X, t)=A T^{2} \exp \left(\frac{e i, h i}{k_{B} T}\right)\left[\exp \left(\frac{q}{k_{B} T}\right) \sqrt{\frac{q E(X, t)}{4}} \quad 1\right]
$$

Where A is the Richardson constant, $j_{\text {Schot }}(X, t)$ refers to the injection current density for electrons or holes, depending on the configuration (under irradiation or post-irradiation) and on the electric field in the material; ${ }_{e}$ and ${ }_{h i}$ are the Schottky injection barrier heights in $[\mathrm{eV}]$.

Depending on the configuration, injection will be possible at one or two electrodes:

- Under electron-beam irradiation: the irradiated face $(\mathrm{x}=\mathrm{d})$ is at a floating potential, and as a first approximation, the electric field on the surface is set to zero while the un-irradiated electrode is grounded $(\mathrm{V}(0, \mathrm{t})=0)$. Hence, injection will only be possible at the grounded electrode, the sign of the injected charge will depend upon the sign of the electric field at this electrode.

- During post-irradiation: the LDPE sample is sandwiched between two electrodes, so charge injection will be possible at both electrodes. The sign of the injected charge at each electrode will depend upon the applied electric field.

\subsection{Model implementation}

Different modules have been tested in order to use the commercial software in the best conditions. Transport of Diluted Species (TDS) has been coupled at first to the Electrostatic module (EC). The model was also developed using the Mathematics module, using a Convection-Diffusion-Equation module, for each species, coupled to the Poisson-Equation module. Artificial diffusion was added to the transport equation developed with mathematics modules. For each model, BDF (Backward Differentiation Formula) solver [11] is used for the time-step. Simulations performed with both 'models' provide mostly the same kind of results, with slightly more smoothing (diffusion) for the TDS module. This is mainly due to numerical scheme used by Comsol to solve the advection equation. We then choose to continue with the mathematics module since customizable.

For each simulation, an initialization step is necessary, as most of the parameters are function of the electric field (mobility, recombination coefficients...).

The domain ( ) studied is in one-dimension, subdivided in $30000, i$ finite elements of length $h_{i}=10 \mathrm{~nm}$ for a domain thickness of $300 \mu \mathrm{m}$. The domain is limited by two boundaries, $\partial$, and 
$\partial{ }_{r}$, positioned at left and right limit of the domain respectively. Figure 3 shows a meshing scheme of the thickness $d$.

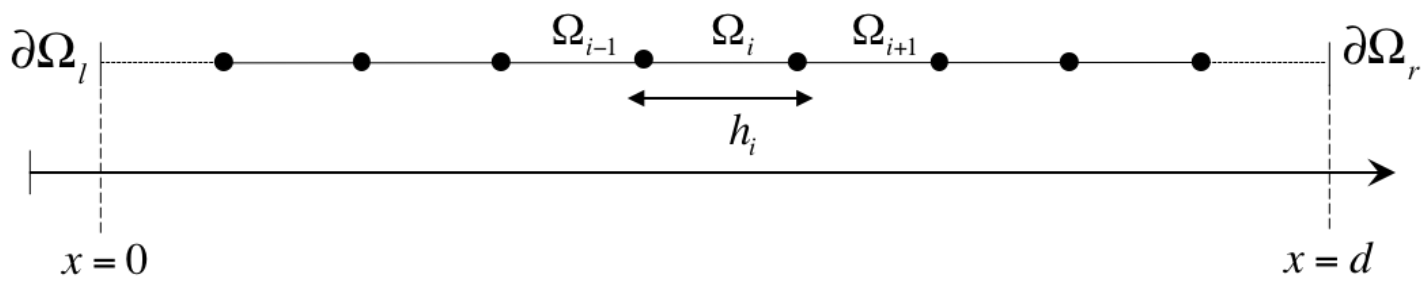

Figure 3. Meshing scheme of the dielectric thickness, representative of the $1 \mathrm{D}$ case. ${ }_{i}$ are the finite elements, $h_{i}$ the element size; $\partial$, and $\partial, r$ are the domain boundaries; $d$ is the domain thickness, i.e. $300 \mu \mathrm{m}$.

\section{Model vs. measurements confrontation}

\subsection{Optimized set of parameters}

In a first attempt, a unique set of parameters was chosen to predict the charge behavior during and after irradiation, for a LDPE material. Transport parameters (hopping barrier height for electrons and holes, detrapping coefficients, deep trap density) are the ones defined for LDPE under electric stress $[12,13]$. Experimentally, different electrode/dielectric contacts have been encountered (Al/LDPE for in-situ PEA measurements, Al/LDPE/SC for 'classic' PEA measurements, Au/LDPE/Au for current measurements). Here SC stands for carbon black-doped polymer used as high voltage electrode in PEA set-up. These differences have been taken into account in the modelling. The injection barrier heights have been optimized for each material ( $\mathrm{Au}, \mathrm{Sc}, \mathrm{Al})$ and for each carrier so as to better fit the experimental results, as it is impossible to use theoretical values. These optimized parameters are reported in Table 1. The value of the electron/hole pairs generation coefficient $\mathrm{G}_{0}$ can be theoretically calculated using the following equation [6]:

$$
G_{0}=\frac{q \times W_{s}}{N \times E_{0} \times r}
$$

Where $W_{s}$ is the energy deposited rate per area unit $\left[\mathrm{W} / \mathrm{m}^{2}\right], \mathrm{N}$ the area under normalized dose curve calculated from Figure 2, $\mathrm{E}_{0}$ the mean energy required to create a pair, estimated at $30 \mathrm{eV}$ by [6] and $\mathrm{r}$ corresponds to the depth of the irradiation region ( $120 \mu \mathrm{m}$ in our case). Taking in consideration our experimental conditions (an electron-beam of energy $80 \mathrm{keV}$ and a current flux of $1 \mathrm{nA} / \mathrm{cm}^{2}$ ), $\mathrm{G}_{0}=983$ $\mathrm{C} \cdot \mathrm{m}^{-3} \cdot \mathrm{s}^{-1}$. This value is however a theoretical estimation, and a sensitivity analysis of this parameter $[8,12]$ has already shown that this value had to be lowered to reproduce the experimental data. Hence, a value of $\mathrm{G}_{0}=8 \mathrm{C} . \mathrm{m}^{-3} \cdot \mathrm{s}^{-1}$ has been retained as the best value to fit all our experimental data. Table 1 summarizes the optimized parameters. The effective Go value can be substantially lower than the theoretically one if most of the created electron-holes pairs produce geminate recombination before being separated and involved in transport/trapping phenomena. It is to note that the trapping coefficients have a different value before and after irradiation. A discussion on this variation will be given in the following paragraphs.

Outputs of the model cannot be directly compared to experimental data, especially space charge profiles obtained by the PEA method. Surface charge densities (capacitive charges and image charges) have been added at the electrodes in the model, and a numerical filter has been applied to the simulated data to lose part of the resolution reached by the simulated profiles and get closer to the experimental data [2]. In the following, this post-treatment will only be added when experimental and simulated space charge data are compared. 
Table 1. Model parameters optimized for simulation of charge generation and transport in electronbeam irradiated and polarized LDPE.

\begin{tabular}{|c|c|c|c|}
\hline Parameter [unit] & \multicolumn{3}{|c|}{ Value } \\
\hline Relative permittivity: $r$ & \multicolumn{3}{|c|}{2.3} \\
\hline Trapping coefficient $\left[\mathrm{s}^{-1}\right]$ & \multicolumn{2}{|c|}{$\begin{array}{c}\text { Before } \\
\text { irradiation }\end{array}$} & $\begin{array}{c}\text { After } \\
\text { irradiation }\end{array}$ \\
\hline Electrons: $\mathrm{B}_{\mathrm{e}}$ & \multirow{2}{*}{\multicolumn{2}{|c|}{$\begin{array}{l}0.1 \\
0.2\end{array}$}} & 0.01 \\
\hline Holes: $\mathrm{B}_{\mathrm{h}}$ & & & 0.02 \\
\hline \multicolumn{4}{|l|}{ Detrapping barrier height $[\mathrm{eV}]$} \\
\hline Electrons: $\phi_{\text {tre }}$ & \multicolumn{3}{|c|}{0.99} \\
\hline Holes: $\phi_{t r h}$ & \multicolumn{3}{|c|}{1.02} \\
\hline Schottky injection barrier height $[\mathrm{eV}]$ & $\mathrm{Au} / \mathrm{LDPE}$ & Al/LDPE & SC/LDPE \\
\hline Electrons: $\phi_{e i}$ & 1.30 & 1.22 & -- \\
\hline Holes: $\phi_{h i}$ & 1.13 & 1.22 & 1.16 \\
\hline \multicolumn{4}{|l|}{ Deep trap density $\left[\mathrm{C} \cdot \mathrm{m}^{-3}\right]$} \\
\hline Electrons: $\mathrm{N}_{0 . \mathrm{et}}$ & & 100 & \\
\hline Holes: $\mathrm{N}_{0 . \mathrm{ht}}$ & & 100 & \\
\hline \multicolumn{4}{|l|}{ Hopping barrier height $[\mathrm{eV}]$} \\
\hline Electrons: $\phi_{a, e}$ & \multicolumn{3}{|c|}{0.713} \\
\hline Holes: $\phi_{a, h}$ & \multicolumn{3}{|c|}{0.636} \\
\hline $\begin{array}{l}\text { Electron/hole pairs generation } \\
\text { coefficient } G_{0}\left[\mathrm{C} \cdot \mathrm{m}^{-3} \cdot \mathrm{s}^{-1}\right]\end{array}$ & \multicolumn{3}{|c|}{8} \\
\hline
\end{tabular}

\subsection{Space charge behavior in non-irradiated LDPE}

The optimized set of parameters has been used to verify the validity of the fluid model under nonirradiation conditions. This will also validate the model developed using the FEM software. Figure 4 shows (a) the experimental and (b) the simulated space charge cartographies obtained for a $300 \mu \mathrm{m}$ thick LDPE polarized under an applied voltage of $12 \mathrm{kV}$ for 1 hour and then short-circuited for $1 \mathrm{~h}$, at $25^{\circ} \mathrm{C}$. Figure $4 \mathrm{c}$ ) also presents the net charge density as a function of position for characteristic times in order to better compare quantitatively the experiment and simulation results. These simulation results have been post-treated to include influence charges (i.e. image charges + capacitive charges) at each electrode. In this case of 'classic' space charge measurement, with Al /LDPE/SC configuration, contacts exist at both electrodes, so injection barrier heights taken for the model are $1.22 \mathrm{eV}$ at the $\mathrm{Al}$ electrode for electrons and $1.16 \mathrm{eV}$ for holes at the SC electrode.

The model seems able to reproduce space charge behaviour observed under polarization/depolarization for a virgin LDPE. The dominance of positive charges over negative ones and the drift of these positive charges, due to electric field are predicted by the model. The arrival time of positive charges at the ground electrode in the simulation $(10 \mathrm{~min})$ is consistent with the experimental one (13 min). No negative charges are apparent during polarization, in the experiment and in the simulation. Under short-circuit, experimentally, there is a fast decrease of the positive charge density, that disappears almost totally at the end of the short-circuit period. Meanwhile, negative charges, previously injected at the ground electrodes but not really observed, are revealed experimentally during short-circuit. In the simulation, negative charges are also present during shortcircuit, but hidden by a high positive charge density, that do not disappear as fast as in the experiment. 

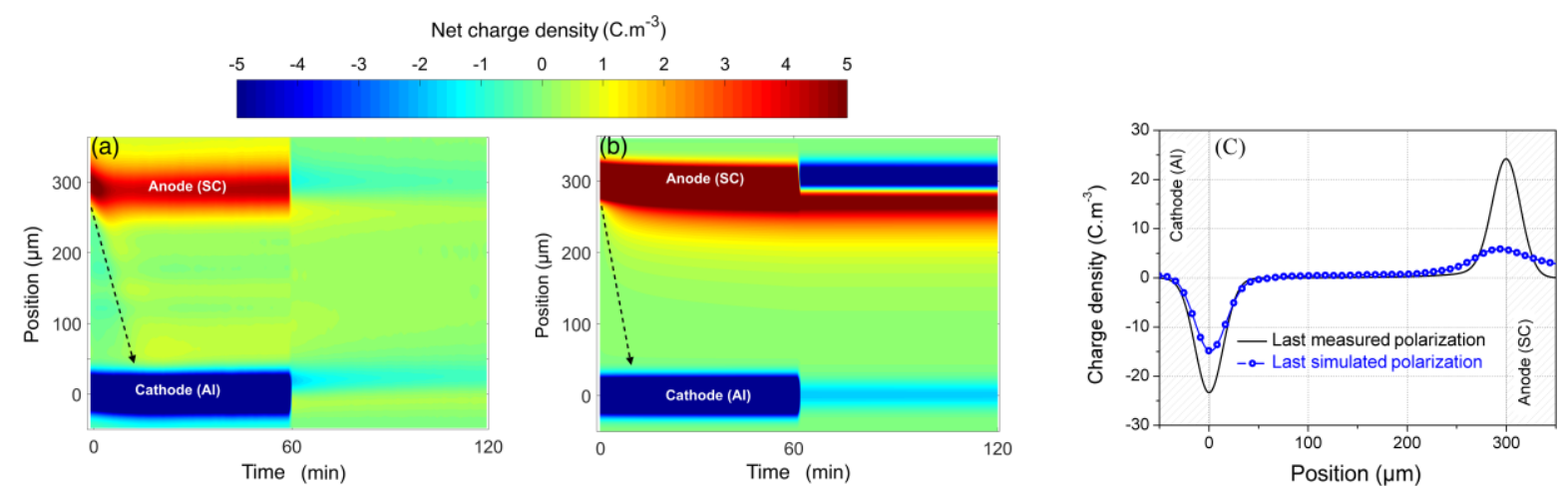

Figure 4. (a and b) Cartographies of space charge density from (a) experimental measurement, and (b) simulation, for a nonirradiated LDPE film of $300 \mu \mathrm{m}$ thickness, at $25^{\circ} \mathrm{C}$. Applied voltage of $+12 \mathrm{kV}$ on the top electrode. (c) Simulated 2D profile for last polarization and depolarization time.

Ici ta figure ne va pas, il me semble qu'il y a un problem au niveau de l'électrode 'anode, car la simulation devrait avoir plus de charge que la mesure, ce qui n'est pas le cas. En plus nous avions dit mesure et simulation pour le dernier temps en poalirzation et en depolarization, donc 4 graphes.

\subsection{Space charge behavior under irradiation}

Figure 5 shows (a) the experimental and (b) the simulated space charge profiles at different irradiation times for a LDPE sample under e-beam irradiation. Under irradiation conditions, the irradiated face of the sample is at a floating potential while the other face is grounded (Al/LDPE contact). Trapping parameters used for this simulation are the one 'before irradiation' of Table 1.

The model is globally able to reproduce the experimental behavior under e-beam irradiation, that is:

- A negative charge peak, with a maximum located at around 90-100 $\mu \mathrm{m}$ from the irradiated face;

- A shift of this peak toward the ground electrode with irradiation time;

- A dominance of negative charges in the profiles: no neat positive charge is detected, even if in the simulation, positive charges are generated by the deposited energy of the beam and can also be injected at the ground electrode.

Nevertheless, some quantitative differences appear between measurement and model. The model shows a maximal peak value $\left(-45 \mathrm{C} \cdot \mathrm{m}^{-3}\right)$ that is more than twice the one measured experimentally ($\left.17 \mathrm{C} . \mathrm{m}^{-3}\right)$. The evolution of the charges peak with the irradiation time also differs between measurement and model. Unlike the measurement, the modeling shows a rapid evolution of the peak maximum between $2 \mathrm{~min}$ and $4 \mathrm{~min}$, before stabilization. These differences could be due to the set of parameters that is not really optimized for this specific experiment. The objective of the project being to globally describe the behavior of space charge over a large range of experiments, the parameters could be better optimized if taking only into account one type of experiment. However, some experimental facts could also explain some of these difficulties to quantitatively reproduce the measurements that are:

- The measurement technique by irradiation periods: It was not possible in this study to measure space charge while the e-beam was on. Hence, space charge measurements are in reality taken between two periods of irradiation (each 2 minutes). One could think that the period during which the e-beam is off could be sufficient to affect the charge distribution within the dielectric. It is to note that an evolution of the space charge measurement installed in the irradiation chamber allows 
now measuring space charge when the e-beam is on [14] but the device was note operating during this study.

- Flashover during irradiation: A second, and most probable explanation for the specific behavior measured experimentally and not predicted by the model is the appearance of discharges during irradiation [7]. These discharges, due to the high potential at the surface of the material, would lead to a rearrangement of the charges inside the material. This could explain the fluctuating peak amplitude observed experimentally with irradiation time. In situ space charge measurements normally show an increase in the peak maximum with the irradiation time [15], as long as the potential is not too high in the vacuum at the surface of the dielectric. This could also explain why the model is not able to predict such behavior.
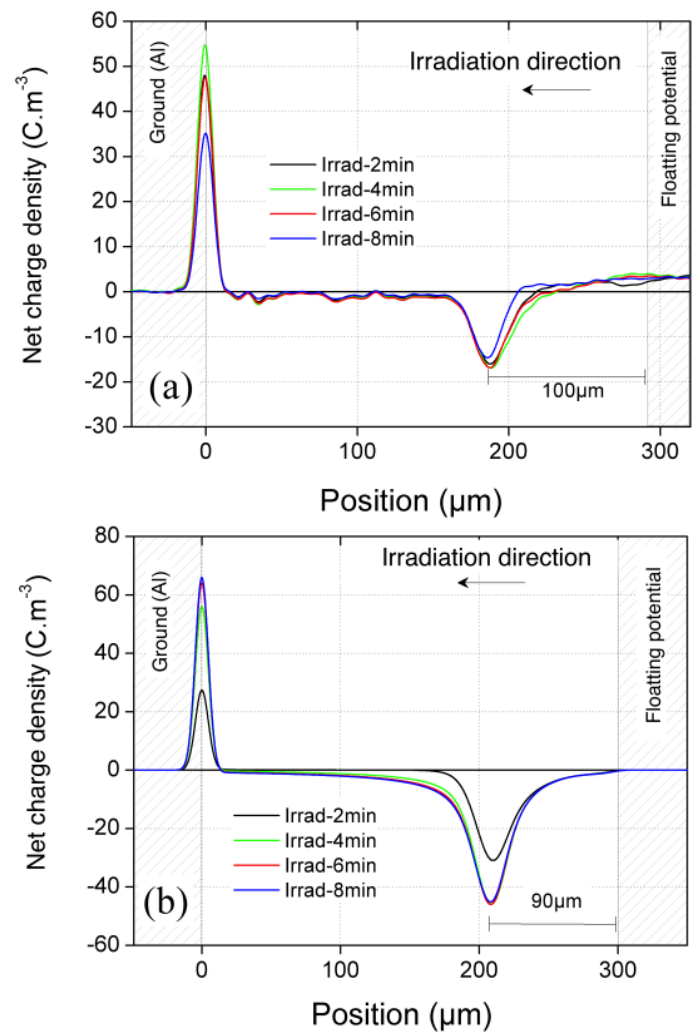

Figure 5. (a) Experimental measurement and (b) simulation of the net charge density vs. position in a $300 \mu \mathrm{m}$ thick LDPE film irradiated by a $80 \mathrm{keV}$ electron-beam with a current flux of $1 \mathrm{nA} / \mathrm{cm}^{2}$. Net charge density taken each 2 min for $8 \mathrm{~min}$. Optimized parameters of Table 1, using trapping coefficient 'before irradiation' and Schottky barrier 'Al/LDPE/Vacuum'.

Unlike in experiment that only measures the net charge density, it is possible to provide the charge profiles for each kind of species in the simulation. Figure 6 a) and b) present the space charge density for each kind of species, being mobile or trapped. Negative charges, mobile and trapped (Figure 6a), are present in the irradiated zone, with two peaks. The first peak is at around $250 \mu \mathrm{m}$, for mobile charges and corresponds to electrons arising from electrons/holes generation (see Figure 2). The second peak is located at around $200 \mu \mathrm{m}$, and is mainly due to trapped electrons. These trapped electrons arise either from deposited electrons or from electron/hole pair generation, that transport across the material and get easily trapped. It is to note that the electric field is responsible for the specific peak of the trapped electrons. This electric field is positive in the zone $[0-200 \mu \mathrm{m}]$ from the grounded electrode (more or less the non-irradiated zone) and becomes small and even null beyond this limit. Hence, electrons located in the non-irradiated zone are easily driven towards the ground electrode, where they can be extracted. On the contrary, electrons at around $200 \mu \mathrm{m}$ have quasi zero mobility and hence stay stuck in this zone. This explains the trapped electron depletion in the middle of the irradiated region. This behavior is enhanced by the field dependent mobility.

Although negative charges are present in a large amount, positive charges are also present next to the irradiation electrode, in a non-negligible amount, of the order of $80 \mathrm{C} / \mathrm{m}^{3}$ (Figure $6 \mathrm{~b}$ ). The positive 
charge peak for mobile and trapped charges is at around $250 \mu \mathrm{m}$, corresponding to the maximal dose. Positive charges are also injected at the ground electrode, but in a smaller amount $\left(<2 \mathrm{C} / \mathrm{m}^{3}\right)$ compared to the charge generated by the electron-beam. Hence, the net charge density, being the sum of positive and negative charges (cf. equation 4), completely masks the presence of positive charges. Both experiments and model show that within a few minutes of irradiation, a nearly steady state situation is reached regarding the net charge distribution (cf. Fig. 5). Detailed analysis of the different kind of species (Figure 6) confirms the feature. Under favorable electric field, it means that the flux of deposited excess electrons is extracted at the ground electrode, and there is an equilibrium between electron-hole pairs generation and charge recombination events. In the region where the deposited energy is maximum, the profiles for mobile electrons and mobile holes are similar because they reflect the continuous generation of electron-hole pairs.
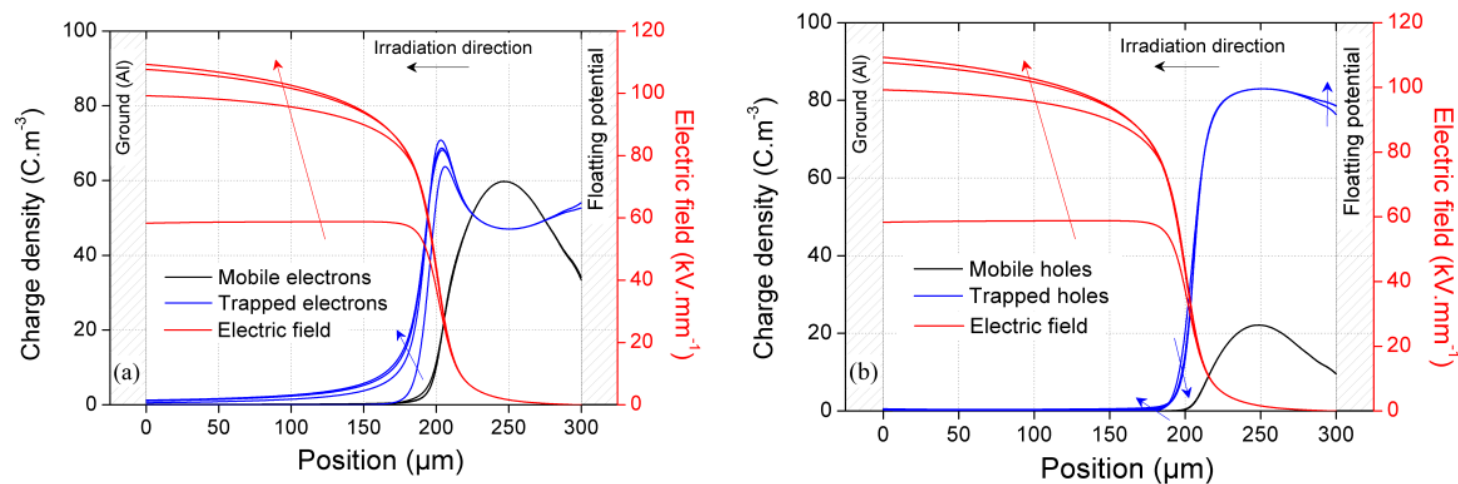

Figure 6. Simulated space charge density (in blue) and associated electric field (in red) vs. position in a $300 \mu \mathrm{m}$ thick LDPE film under irradiation by a $80 \mathrm{keV}$ electron-beam with a current flux of $1 \mathrm{nA} / \mathrm{cm}^{2}$ for 2, 4, 6 and $8 \mathrm{~min}$. Arrows indicate increasing irradiation time. (a) Mobile and trapped electrons; (b) mobile and trapped holes.

\subsection{Space charge behavior during post-irradiation}

\subsubsection{Polarization/depolarization protocol}

For probing the behavior of LDPE in post-irradiation, the same kind of sample of thickness $300 \mu \mathrm{m}$ has been irradiated during 10 minutes in the same irradiation conditions as previously, then removed from the irradiation chamber and inserted at room temperature in a 'classic' PEA set-up. Details of the experiments can be found in [1]. Before any voltage is applied, the space charge measurements are performed during $5 \mathrm{~min}$ under short-circuit conditions in order to check the presence of electrons deposited by the electron beam during irradiation. A voltage of $12 \mathrm{kV}$ is then applied for 1 hour, followed by short-circuit period of 1 hour, as for the non-irradiated sample in section 3.2. Figure 7a) presents the measured space charge density as a function of time and position in the sample for the whole protocol (5 min of short-circuit, 1hour of polarization, 1 hour of depolarization). It can be observed that during the first 5 min under short circuit, a negative charge is observed at around 100 $\mu \mathrm{m}$ from the previously irradiated face, which corresponds to the deposited electrons by the beam. The peak maximum is however of the order of -2 to $-3 \mathrm{C} / \mathrm{m}^{3}$ in this case, i.e. much lower compared to the in situ measurement of Figure 5a), where the peak maximum was of the order of $-17 \mathrm{C} / \mathrm{m}^{3}$. This decrease of the charge density certainly comes from partial neutralization during the transfer from the irradiation chamber under vacuum to atmospheric pressure. However, the physical processes at play are not yet understood. To account for this charge decrease in the model, the simulated space charge densities obtained in Figure $5 b$ ) have been divided by 15 , to obtain the same charge density as the one measured in the 'classic' PEA set-up. Figure 7b) presents the simulated space charge density profiles as a function of time for the whole protocol (5 min of short-circuit, 1 hour of polarization and 1 hour of depolarization), and can be directly compared to experimental data of Figure 7a).

- During short-circuit before polarization, the presence of the negative charges deposited by the electron-beam is well reproduced by the model at about $100 \mu \mathrm{m}$ from the irradiated surface. The simulated amplitude is comparable to the experimental one. However, the positive charges observed 
experimentally are not reproduced by the simulation. Figure 8a presents the simulated charge density, with no image charge and no attenuation of the signal, as a function of the sample position for different short-circuit times. It is possible to observe next to each electrode the presence, in a small amount, of positive charges, that appear to be injected at both electrodes, considering the electric field distribution (Figure 8b). At the right electrode, the net charge at $t=0 \mathrm{~s}$ is negative, but decreases with short-circuit, to become positive after 5 min under short-circuit. This is the result of positive charge injection, as the electric field is favourable, and negative charge extraction. These charges are however not visible once the signal treatment is applied to the simulated profiles.

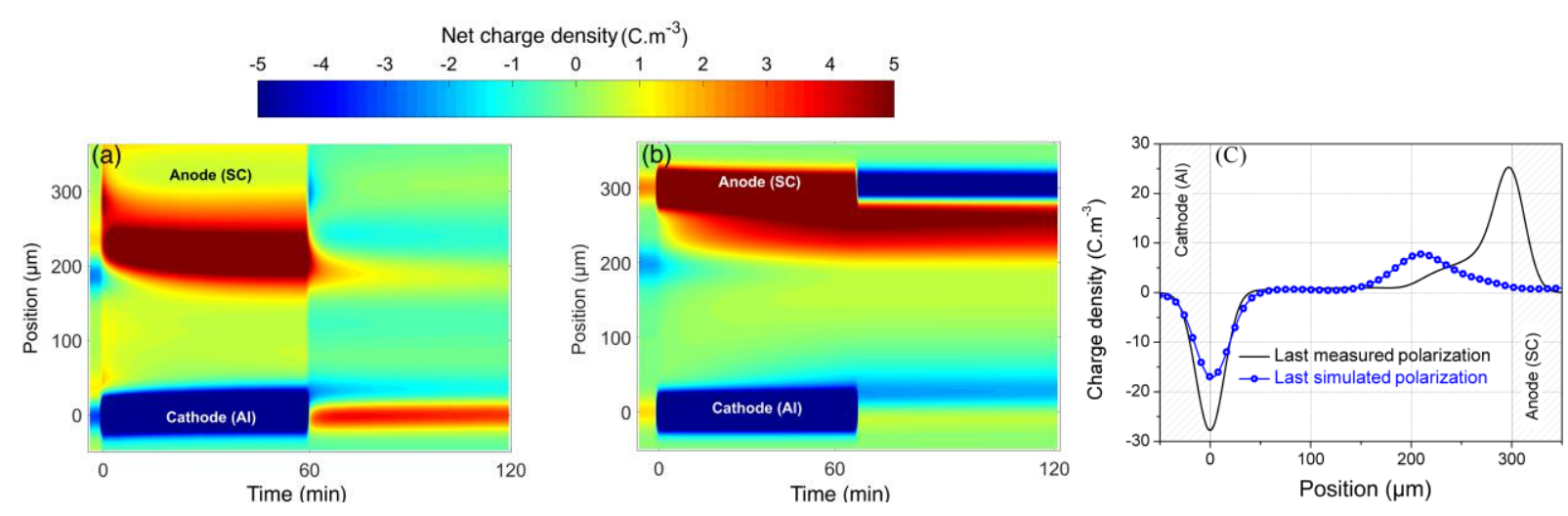

Figure 7. (a and b) Cartographies of space charge density for an e-beam irradiated LDPE film of $300 \mu \mathrm{m}$ thickness, at $25^{\circ} \mathrm{C}$. Short-circuit for 5 minutes; then voltage of $+12 \mathrm{kV}$ applied on the top electrode (i.e. electric field of $40 \mathrm{kV} / \mathrm{mm}$ ) for one hour; and short-circuit for one hour. Irradiated zone next to the top electrode. (a) Experimental measurement; (b) simulation. Optimized parameters from Table 1, using trapping coefficient 'After irradiation' and Schottky barrier 'Al/LDPE/SC'. (c) Simulated 2D profile for last polarization and depolarization time.

Ici ta figure ne va pas, il y en a un en court circuit (simulation) et l'autre sous tension. En plus nous avions dit mesure et simulation pour le dernier temps en poalirzation et en depolarization, donc 4 graphes.
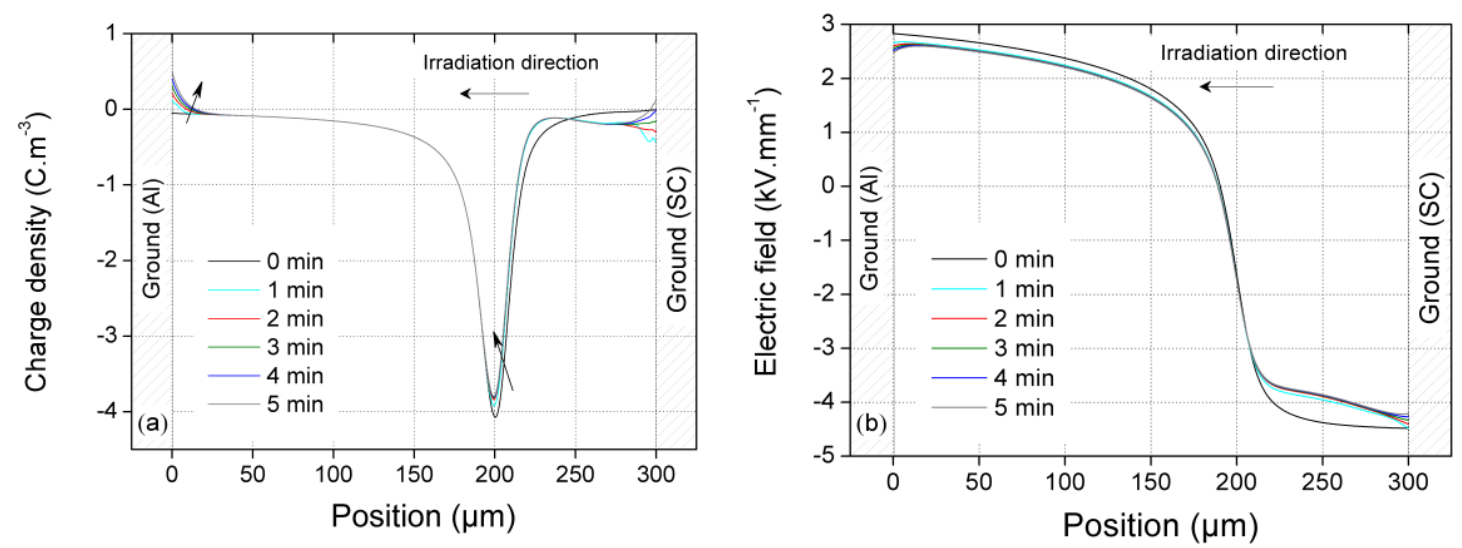

Figure 8. Simulated (a) net charge density and (b) associated electric field vs. thickness of the sample, during short-circuit in air after irradiation (and before polarization).

- During polarization $(1 h)$ : the main experimental characteristics are reproduced by the model. The massive generation of positive space charge region, observed experimentally around the irradiated zone, seems well reproduced by the model. The build-up of hole charges cloud is also almost instantaneous in the model as soon as the voltage is applied. It is however difficult only with the net charge density to understand the origin of this positive charge appearance. To unravel the possible 
origins of this phenomenon, simulated holes injection and electrons extraction current densities have been plotted as a function of time, for a non-irradiated (Figure 9a) and an irradiated (Figure 9b) sample. Although the hole injection flux is higher by two decades in the case of an irradiated sample compared to a non-irradiated one, the main difference arises from the electrons extraction flux. It is quasi null $\left(10^{-22} \mathrm{~A} / \mathrm{m}^{2}\right)$ in the non-irradiated case, while it is of the order of $10^{-8}$ to $10^{-10} \mathrm{~A} / \mathrm{m}^{2}$ for the irradiated case. The current density equation (6) for electron extraction is function of the charge density, the mobility, and the electric field. For hole injection, the current density is mainly function of the electric field and the temperature as variables. The electric field at the anode is of the order of $44 \mathrm{kV} / \mathrm{mm}$ at the beginning of polarization (Figure 10). It is hence 10\% higher than the applied electric field $(40 \mathrm{kV} / \mathrm{mm})$. This increase of the electric field can explain the increase in the hole injection flux, but not the one of the electron extraction, which is mainly due to the high electron density at the anode. Moreover, Figure 11 compares the current density as a function of position within the sample for electrons and holes after one minute of polarization in the case of a nonirradiated sample (Figure 11a) and in the case of an irradiated one (Figure 11b). The main difference for electrons arises in the irradiated zone, where the current density is quasi null in the case of the non-irradiated sample. On the contrary, the current density for electrons in the case of an irradiated sample is high, i.e. the electron transport is also high within this region, driving the electrons towards the anode, where they are extracted. The current density is also higher for holes in the whole dielectric, i.e. transport also plays a non negligible role. Hence, the high transport and extraction of electrons at the anode coupled to a high rate of injection and transport of holes implies the specific behavior reproduced by the simulation. At the bottom electrode, it is possible to observe, experimentally and in the simulation, the injection of negative charges during polarization.
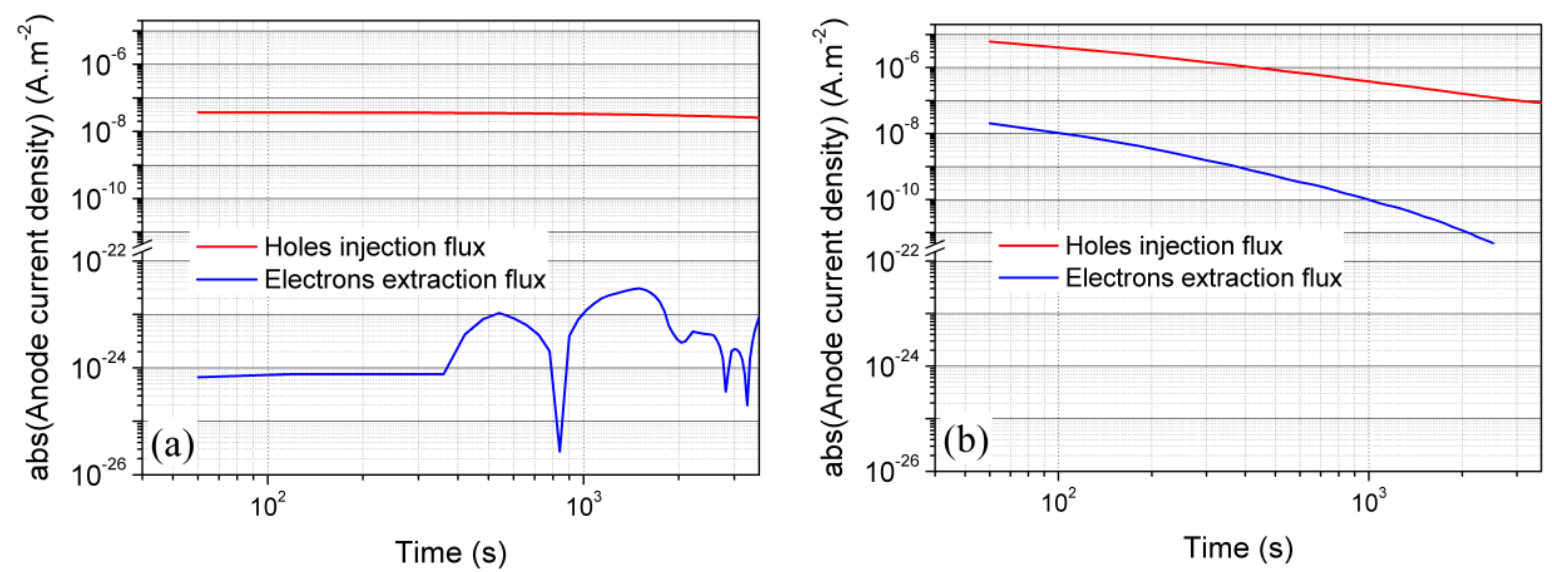

Figure 9. Simulated current densities of electrons and holes at the anode as a function of the polarization time. (a) Unirradiated sample and (b) Irradiated sample. 


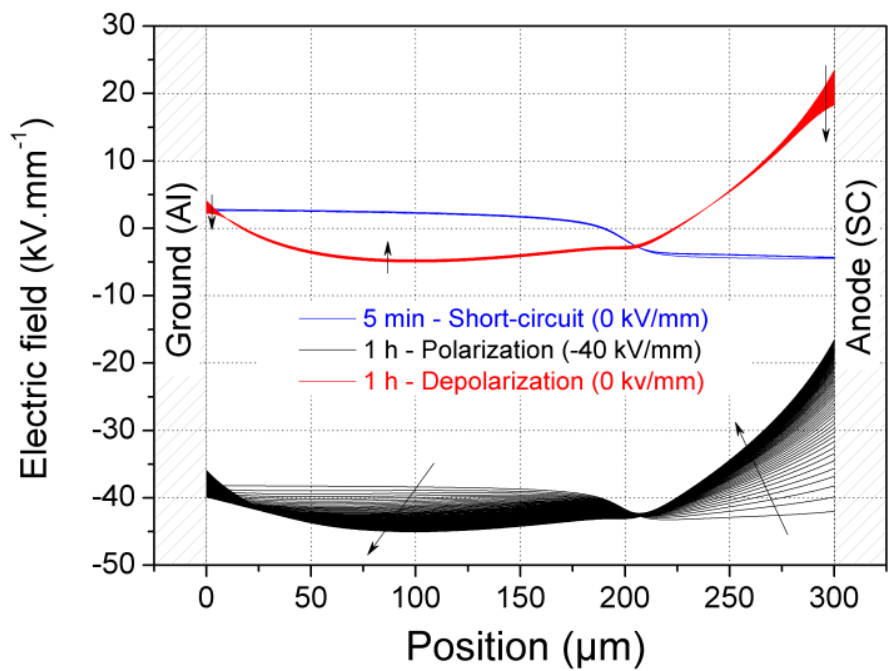

Figure 10. Electric field associated to the simulated net charge density from figure 7, for an irradiated LDPE sample of $300 \mu \mathrm{m}$ thickness, at $25^{\circ} \mathrm{C}$. Short-circuit for 5 minutes; voltage of $+12 \mathrm{kV}$ on the top electrode (i.e. electric field of 40 $\mathrm{kV} / \mathrm{mm}$ ) for one hour; and short-circuit for one hour. Optimized parameters from Table 1, using trapping coefficient 'After irradiation' and Schottky barrier 'Al/LDPE/SC'.
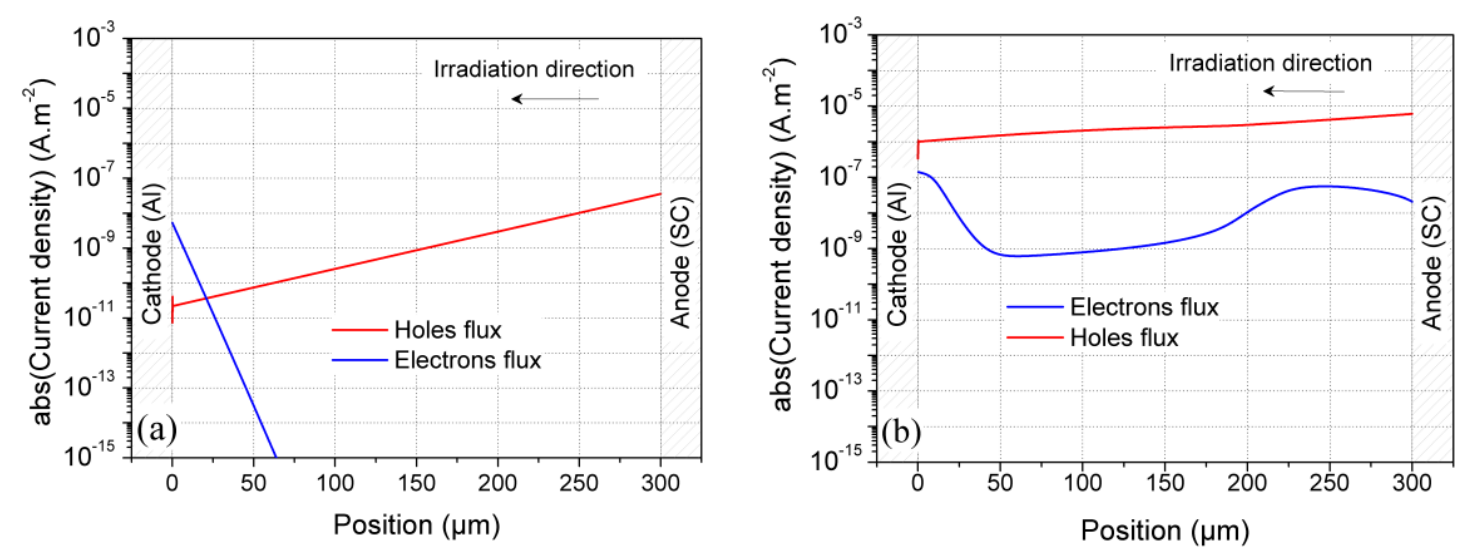

Figure 11. Simulated current densities of electrons and holes after 1 min under polarization as a function of the thickness of the sample. (a) Un-irradiated sample and (b) Irradiated sample.

- During depolarization $(1 \mathrm{~h})$ : The decrease of positive charges stored near the top electrode and the one of negative charges measured at the bottom electrode are reproduced by the model, although the decrease is slower in the case of the simulation. The simulated electric field, presented in Figure 10 during depolarization, allows positive charge extraction at the top electrode, and negative charge extraction at the bottom electrode, that decreases the global net charge density in the material. This is however not enough to reproduce the experimental behavior. This was already the case for simulations performed on metal/insulation/metal system under various DC fields $[2,16]$, where the release of charges was always slower in the simulations compared to the experiment. Either charges are more mobile than what is predicted by the model, or other processes are at play, that the model does not take into account at the moment.

\subsubsection{Current during step increase of Voltage}

The last protocol used to validate the model and the parameters is ex-situ current measurements, performed on non-irradiated and on irradiated LDPE samples. The protocol of voltage application consisted in increasing the applied electric field by steps of $5 \mathrm{kV} / \mathrm{mm}$ up to $100 \mathrm{kV} / \mathrm{mm}$ with resting time of 10 min for each step. The current was measured with positive voltage applied on the irradiated face. The sample (irradiated and non-irradiated) is sandwiched between two gold electrodes. This implies in the modeling to consider an injection barrier height relevant to a 'Au/LDPE/Au' contact. 
Figure 12 shows a comparison of the measured and simulated current density as a function of electric field for both irradiated and non-irradiated LDPE films.

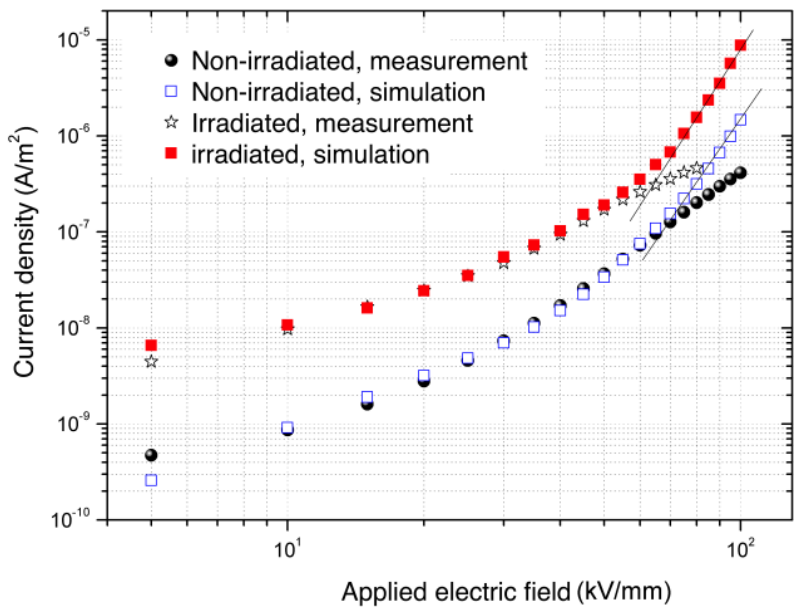

Figure 12. Experimental and simulated current density of Au/LDPE/Au samples as a function of the applied electric field. Step increase of voltage protocol. Optimized parameters are given in Table 1.

Globally, for electric fields below $70 \mathrm{kV} / \mathrm{mm}$ for non-irradiated and $60 \mathrm{kV} / \mathrm{mm}$ for irradiated samples, the simulation results are consistent compared to the measured ones. The currents measured/simulated for the non-irradiated case are more than one decade below the ones measured/simulated for the irradiated case. This is mainly due to charges that are present in the irradiated sample, and hence contribute to a current. This is not the case for a non-irradiated sample. This difference between irradiated/non-irradiated samples disappears slowly when the electric field is increased. It seems as if at high electric field, the irradiated LDPE behaves like a virgin LDPE. This should come from the fact that charges, injected at the electrode, overcome the charge density already present in the bulk due to irradiation. The other reason for the difference in simulated current between irradiated and nonirradiated samples is the change in trapping coefficient. The experimental currents follow a space charge limited current - SCLC shape since at fields above $70 \mathrm{kV} / \mathrm{mm}$, the slope of the $\mathrm{J} / \mathrm{E}$ curve decreases. This feature is not reproduced in the simulations. The simulation curves are dominated by the hopping process mainly, that drastically increases the current density as the field increases. The model is not able to reproduce this decrease of the current-field slope when the field increases. This has already been explained in [17], where the third region corresponding in the literature to a trap filled region, is achieved for fields higher than what is observed experimentally. This shift towards the higher electric fields might be due to the fact that physical processes, such as trapping, are not well described in the actual fluid model.

\section{Discussion}

The model developed is able to reproduce most characteristics observed on both virgin and e-beamirradiated LDPE samples. However, some care has to be taken regarding the model hypotheses and the optimized set of parameters:

- It has been observed that the model tends to overestimate the charge density under irradiation and during depolarization for ex-situ measurements. This has been observed in several cases study published in the literature [2,16]. Typically, the simulated charge profile decays slower than the experimental one. This means that there is a lack of understanding in some specific physical processes during depolarization.

- Trapping coefficients have been modified after irradiation, to better fit the experimental data, being space charge measurements or current measurement. Using the trapping coefficients previously optimized [2], the simulation results were not as good as the one obtained with the parameters presented in Table 1, especially for the step increase of voltage for the irradiated case. It is to note that this is one of the most difficult experiments to reproduce, as only an integrated value (current over the 
thickness of the sample) is available, that takes into account the previous polarization steps and so the charge distribution in the bulk. The lowering of these trapping coefficients could mean that the electron irradiation would change the trap distribution, directly linked to the chemical structure of the material. Physico-chemical measurements performed by infrared spectroscopy on irradiated samples [1] show an increase of the $\mathrm{CH}_{3}$ bonds and a decrease of the $\mathrm{CH}_{2}$ bonds for the irradiated samples (i.e. chain scissions), as well as an increase of the $\mathrm{C}=\mathrm{O}$ bonds. This would however lead to the creation of deep traps, so the trapping coefficient should increase. This is not what has been found in the present study. One of the hypotheses could be that traps, even in higher concentration and/or higher depth, would be filled faster with the electron beam, leading to an early trap-filled limit behavior. The fact that a much smaller $G_{\theta}$ value than caleulated from Eq. (12) has to be used in the model may contain such redistribution of trap oceupancy. Only shallower traps, either created by the e-beam or exhibiting a smaller capture cross-section, could then be available for transport when applying a DC field. Anyway, both charging and change in the trap distribution (i.e. variation of the trapping coefficients in the model) were the processes dominating the charge pattern in the simulation results, and it is difficult to segregate one from the other. This also raises the question of the relevance of a unique level of deep traps as hypothesis for the fluid model.

- In the present study, three kinds of electrode/LDPE interfaces have been used experimentally (Al, $\mathrm{Au}$, and SC electrodes). It has been necessary to use in the simulations different barrier heights to optimize the simulation results. This means that the contact type plays a key role in the simulations and in the experimental behaviour of dielectrics at high fields. This has already been highlighted in other experimental or theoretical works (ref).

\section{Conclusion}

A fluid bipolar charge transport model has been developed under COMSOL Multiphysics, and adapted to electron beam irradiation under vacuum conditions. It proposes an evolution in the physical hypotheses by including a hopping mobility and recombination parameters that are dependent upon charge mobility. Simulation results under different conditions (in situ, ex-situ space charge measurements, current measurements using a step increase of voltage) globally show a good agreement with experimental data. The specific behavior observed for space charge measurements and current measurements for irradiated material seems to be mostly explained by transport of charges during irradiation (a form of delayed radiation induced conductivity). However, not everything could be modelled relying on same physical parameters for irradiated and non-irradiated material. Here experiment and simulation were reconciled considering a lower deep trapping coefficient after irradiation, which could be an apparent consequence of deep trap filling or shallower traps formation as well. FTIR measurements revealed some modifications of material structure. However, at present we did not establish straightforward link between those possible structural changes and changes in the trap distribution suggested by the transport model.

Hence, some specific points need to be highlighted for future model development. Even for a simple material as LDPE, the relevance of a unique level of deep traps is in question, as it seems that e-beam irradiation either creates traps in the material or leads to trapping levels not available by classical processes. The trapping model could also be at the origin of some divergence of the simulation results compared to experimental data. It has also been highlighted that injection remains one of the key process in space charge behavior in LDPE. The use of electron beam irradiation, coupled with dielectric and physico-chemical post-polarization measurements, allows a better view of the strengths and weaknesses of the model developed. This, coupled with optimization tools, will lead to a better description of the physical processes necessary to describe the space charge behavior in LDPE, under a given constraint.

\section{References}

[1] Banda M E, Griseri V, Teyssedre G and Le Roy S 2018 J. Phys. D: Appl. Phys. 4 155303-13

[2] Le Roy S, Teyssedre G, Laurent C, Montanari G C and Palmieri F 2006 J. Phys. D: Appl. Phys. 39 1427-36

[3] Hoang A T, Serdyuk Y V. and Gubanski S 2016 Polymers 8 103-19 
[4] Min D, Wang W and Li S 2015 IEEE Trans. Dielectr. Electr. Insul. 22 1483-91

[5] Ferreira G L and De Figueiredo M T 2003 IEEE Trans. Dielectr. Electr. Insul. 12 137-47

[6] Sessler G M, De Figueiredo M T and Leal Ferreira G F 2004 IEEE Trans. Dielectr. Electr. Insul. 11 192-202

[7] Nguyen X T, Griseri V and Berquez L 2013 Proc. Int. Conf. Electrical Insulation and Dielectric Phenomena (Shenzhen, China) pp. 226-69

[8] Le Roy S, Baudoin F, Griseri V, Laurent C and Teyssedre G 2012 J. Appl. Phys. 112023704

[9] Le Roy S, Baudoin F, Griseri V, Laurent C and Teyssedre G 2010 J. Phys. D: Appl. Phys. 43 315402-11

[10] Briesmeister J F 2000 Version 4C, LA-13709-M, Los Alamos National Laboratory, 2

[11] Press W, Teukolsky S, Vetterline W T and Flannery B P 2007 Cambridge University Press, (Cambridge, UK) 12-13

[12] Banda M E, Griseri V, Teyssedre G and Le Roy 2018 Proc. IEEE Int. Conf. on Dielectrics, (Budapest, Hungary), 1-4

[13] Le Roy S, Teyssedre G and Laurent C 2016 IEEE Trans. Dielectr. Electr. Insul. 23 2361-7

[14] Riffaud J, Griseri V and Berquez, L 2016 IEEE International Conference on Dielectrics 1163 6

[15] Perrin C, Griseri V and Laurent C 2008 IEEE Trans. Dielectr. Electr. Insul. 15, 958

[16] Matsui K, Tanaka Y, Takada T and Maeno T 2008 IEEE Trans. Dielectr. Electr. Insul. 15, 84150

[17] Baudoin F, Le Roy S, Teyssedre G and Laurent C 2008 J. Phys. D: Appl. Phys. 41 02530615

[C1] Le Roy S, Teyssedre G, and Laurent C 2016 IEEE Transactions on Dielectrics and Electrical Insulation 23 2361-67

[C2] Mott N F and Davis E A 2012 Electronic processes in non-crystalline materials. Oxford university press.

[C3] Hybertsen M S and Louie S G 1986 Physical Review B 345390

[C4] Tuncer E, Serdyuk Y V and Gubanski S M 2002 IEEE Transactions on Dielectrics and Electrical Insulation, 9 809-28. 